\title{
Hongos tremeloides (Heterobasidiomycetes) de la Reserva de la Biosfera de Calakmul, Campeche, México
}

\section{Tremelloid fungi (Heterobasidiomycetes) from Calakmul Biosphere Reserve, Campeche, Mexico}

Sigfrido Sierra ${ }^{1 凶}$, Ibeth Rodríguez-Gutiérrez ${ }^{1}$, Laura Adriana Izquierdo-San Agustín ${ }^{1}$, Sandra Castro-Santiuste ${ }^{1}$, Joaquín Cifuentes ${ }^{2,3}$ y Lilia Pérez-Ramírez ${ }^{3}$

${ }^{1}$ Laboratorio de Taxonomía de Hongos Tremeloides, Facultad de Ciencias, Universidad Nacional Autónoma de México. Ciudad Universitaria, Apartado postal 70181, 04510 México, D. F., México.

${ }^{2}$ Laboratorio de Biodiversidad y Biogeografia Ecológica de Hongos, Facultad de Ciencias, Universidad Nacional Autónoma de México. Ciudad Universitaria, Apartado postal 70181, 04510 México, D. F., México.

${ }^{3}$ Herbario FCME, Facultad de Ciencias, Universidad Nacional Autónoma de México. Ciudad Universitaria, Apartado postal 70181, 04510 México, D. F., México.

\ssg@fciencias.unam.mx

Resumen. Se registran 7 especies de hongos tremeloides de la Reserva de la Biosfera de Calakmul: Auricularia cornea Ehrenb., A. delicata (Fr.) Henn., A. mesenterica (Dicks.) Pers., Dacryopinax elegans (Berk. et M.A. Curtis) G.W. Martin, D. spathularia (Schwein.) G.W. Martin, Tremella wrightii Berk. et M.A. Curtis y Tremelloscypha gelatinosa (Murrill) Oberw. et K. Wells. Todas son registros nuevos para la reserva. Auricularia cornea y T. gelatinosa son nuevos registros para el estado de Campeche.

Palabras clave: biodiversidad, macromicetos, selva tropical.

\begin{abstract}
Seven species of tremelloid fungi are recorded from Calakmul Biosphere Reserve: Auricularia cornea Ehrenb., A. delicata (Fr.) Henn., A. mesenterica (Dicks.) Pers., Dacryopinax elegans (Berk. et M.A. Curtis) G.W. Martin, D. spathularia (Schwein.) G.W. Martin, Tremella wrightii Berk. et M.A. Curtis and Tremelloscypha gelatinosa (Murrill) Oberw. et K. Wells. All are new records for the reserve. Auricularia cornea and T. gelatinosa are new records for Campeche state.
\end{abstract}

Key words: biodiversity, macrofungi, tropical forest.

\section{Introducción}

Los hongos son uno de los grupos de organismos con mayor diversidad; se calcula que en el mundo debe de haber por lo menos un millón y medio de especies (Hawksworth, 1991; 1997; Hawksworth et al., 1995). La posición biogeográfica de México ha propiciado que posea una de las biotas más ricas, donde los hongos no son la excepción. Sin embargo, el conocimiento de la micobiota en este país, al igual que en el resto de los países, es insuficiente (Cifuentes, 1996; Guzmán, 1998), sólo se conoce en parte y se limita principalmente a ciertas regiones en los estados del centro. Aunque cada día se incrementan los estudios fungísticos de otras zonas, éstos son parciales y más aún los que se refieren a las zonas tropicales.

Recibido: 28 marzo 2011; aceptado: 10 agosto 2011
Pocos son los trabajos micológicos para la península de Yucatán en general (Chio y Guzmán, 1982; Guzmán, 1983, 2003, 2004; Pérez-Silva et al., 1992a) y en menor medida los que estudian el estado de Campeche (Guzmán y Madrigal, 1967; Herrera et al., 2005). Para esa entidad se han registrado 81 especies de macromicetos y 5 de líquenes, de las cuales sólo 8 se citan para la Reserva de la Biosfera de Calakmul (RBC).

Respecto a los hongos tremeloides, también es escaso el conocimiento que de ellos se tiene. Entre los estudios enfocados a éstos, están los de Lowy (1965), García-Romero et al. (1970), Lowy y Guzmán (1979), Pérez-Silva et al. (1992a), Sierra y Cifuentes (1993; 1998; 2005) y Sierra et al. (2008). Dentro del uso que se les da a algunos representantes de este grupo se registra su comestibilidad, como así lo citan Ruán-Soto et al. (2007, 2009) y Sierra et al. (2008). De la península de Yucatán se han registrado 16 hongos tremeloides (Guzmán y Madrigal, 1967; Chio y Guzmán, 1982; Guzmán, 1983, 
2003, 2004; Pérez-Silva et al. 1992; Sierra y Cifuentes, 2009) y Roberts (2008) describe 28 especies de Heterobasidiomycetes de Belice.

La zona de la RBC, en el estado de Campeche, es un importante corredor biológico que permite el desplazamiento de los organismos en ambas direcciones, entre las regiones subxéricas del norte de la península de Yucatán y las zonas de clima subhúmedo y húmedo del sur. Se localiza al sureste del estado en el municipio de Calakmul, limitando al este con el estado de Quintana Roo y al sur con la República de Guatemala. Las coordenadas extremas en que se ubica la RBC son $19^{\circ} 15^{\prime}$ y $17^{\circ} 45^{\prime} \mathrm{N}$ y $90^{\circ} 10^{\prime}$ y $89^{\circ} 15^{\prime} \mathrm{O}$ (INE, 1999). Comprende una extensión total de 723185 ha, con 2 zonas núcleo, que en su conjunto comprenden un área total de 248260 ha y una zona de amortiguamiento de 474924 hectáreas. La cruzan 2 ejes carreteros; el primero, oriente-poniente, corresponde a la carretera federal Escárcega-Chetumal que la divide en 2 fracciones y el segundo eje se divide en 2 tramos; uno es la carretera estatal Dzibalchen-X'pujil, que corre de norte a sur y atraviesa las zonas núcleo y de amortiguamiento de la porción norte, y el otro va de X'pujil hacia Arroyo Negro, bordeando la RBC (INE, 1999). La de Calakmul es la mayor reserva de bosque tropical en México, con características climatológicas, edafológicas y de vegetación muy particulares. Es una mezcla de selvas altas y medianas con selvas bajas temporalmente inundables y vegetación acuática. La fauna de la zona es relevante por su representación de mamíferos. Aunque no cuenta con vertebrados endémicos, contiene un número alto de especies consideradas raras, amenazadas o en peligro de extinción (INE, 1999). Aunado a esto, en la reserva se localiza uno de los agrupamientos de zonas arqueológicas de la cultura maya más sobresalientes del país (INE, 1999).

\section{Materiales y métodos}

Durante los años 2001 a 2006, se recolectaron 22 ejemplares de hongos tremeloides provenientes de 9 localidades de Calakmul, Campeche, todas pertenecientes a la $\mathrm{RBC}$, que se numeran y especifican a continuación; todas son del municipio de Calakmul. De la localidad 3 a la 9, el kilometraje es de la desviación a Calakmul, carretera Escárcega-Chetumal.

1. Cerca de la Zona Arqueológica de Calakmul, 1806'35" N y $89^{\circ} 48^{\prime} 17$ ' O. 2. Km. 6-7 de la desviación a la comunidad 20 de Junio, carr. Dzibalchen-X'pujil, 19³7'15" N y $89^{\circ} 21$ '54" O. 3. Km. 21, $18^{\circ} 20^{\prime} 04^{\prime \prime}$ N y $89^{\circ} 52^{\prime} 10^{\prime \prime}$ O. 4. Km. $24,18^{\circ} 18^{\prime} 42^{\prime \prime} \mathrm{N}$ y $89^{\circ} 51$ '16” O. 5. Km. 25.5, $18^{\circ} 18^{\prime} 02^{\prime \prime} \mathrm{N}$ y $89^{\circ} 50^{\prime} 47^{\prime \prime}$ O. 6. Km. 26, $18^{\circ} 17^{\prime} 48^{\prime \prime}$ N y $89^{\circ} 50^{\prime} 37^{\prime \prime}$ O. 7 . Km. $27,18^{\circ} 17^{\prime} 20^{\prime \prime}$ N y $89^{\circ} 50^{\prime} 19^{\prime \prime}$
O. 8. Km. $28,18^{\circ} 16^{\prime} 57^{\prime \prime} \mathrm{N}$ y $89^{\circ} 49^{\prime} 53^{\prime \prime}$ O. 9. Km. 39, $18^{\circ} 14^{\prime} 08^{\prime \prime} \mathrm{N}$ y $89^{\circ} 47^{\prime} 49^{\prime \prime} \mathrm{O}$.

El material se encuentra depositado en el Herbario de la Facultad de Ciencias (FCME) de la UNAM. Los ejemplares se estudiaron siguiendo las técnicas micológicas propuestas por Martin (1952), Lowy (1971), Cifuentes et al. (1986) y Sierra (1992). Se utilizaron las guías de colores de Munsell (Munsell Color Co., 1975), Methuen (Kornerup y Wanscher, 1978) y Küppers (1979).

\section{Descripciones}

En el presente estudio se registran 7 especies de hongos tremeloides, como resultado de las exploraciones realizadas en la RBC. Se citan por primera vez para el estado de Campeche A. cornea y T. gelatinosa y todas son registros nuevos para la reserva.

Tremella wrightii Berk. et M. A. Curtis, J. Linn. Soc. Bot. 10:341 (1868) (Fig. 1A)

Basidioma hasta $40 \mathrm{~mm}$ de ancho y $30 \mathrm{~mm}$ de alto; globoso-lobulado, lóbulos aplanados en la parte superior; gelatinoso-cartilaginoso, superficie húmeda-viscosa; de color café-rojizo (Küppers A90 M99 C80), se obscurece al secarse. Hifas de 2-3 $\mu \mathrm{m}$ de ancho en el contexto e irregularmente infladas en el subhimenio, de hasta 5-6 $\mu \mathrm{m}$; con fíbulas. Anastomosis presente en las hifas subbasidiales del himenio. Basidios de 10-12 $\mu \mathrm{m}$ de ancho, de 4 células, subglobosos a elipsoides, con septos verticales. Basidiosporas de 5-7 × 4-6 $\mu \mathrm{m}$, elipsoides, lisas, hialinas, apéndice hilar conspicuo

Resumen taxonómico.

Hábitat y hábito. Lignícola; sobre restos de madera no identificada, gregario.

Material estudiado. CAMPECHE: Loc. 4, Vite-Garín 37, 10-sep-2004 (FCME 20862).

Comentarios taxonómicos. Esta especie es similar a Tremella foliacea Pers. pero T. wrightii presenta hifas más anchas e irregularmente infladas, así como anastomosis en las hifas sub-basidiales, no observadas en $T$. foliacea. Los lóbulos foliáceos o esparasoides de $T$. foliacea son también distintos a los lóbulos inflados de T. wrightii (Lowy, 1971). Lowy (1971) y Guzmán (2003) mencionaron que en $T$. wrightii, los lóbulos además de ser huecos son aplanados en la parte superior. Roberts y de Meijer (1997) describieron basidiomas de color más claro y con protuberancias digitiformes que no se observaron en el material de Campeche. Se ha registrado de los estados de Campeche y Quintana Roo (Chio y Guzmán, 1982; Guzmán, 2003).

Tremelloscypha gelatinosa (Murrill) Oberw. et K. Wells, in Wells et Oberwinkler, Mycologia 74(2):325 (1982) (Fig. 1B) 
Basidiomas flabelados a infundibuliformes; de consistencia esponjosa. Píleo de 15-80 mm en diámetro; fibriloso-algodonoso; amarillo pálido a gris (Munsell 2.5 Y8/4 a 8/3, Methuen 8B1). Estípite pobremente desarrollado, crece alrededor de la parte basal de tallos de plantas; superficie algodonosa; amarillo pálido a blanco (2.5Y8/4). Contexto esponjoso, amarillo pálido (2.5Y8/3). Himenio liso; color café oscuro (7.5YR3/3 a $3 / 4)$, café rojizo (7E8), hasta café vináceo (9F7), o blanco a rosado (8A1 a 8B3). Basidios de 16.5-18.5 × 10-11.5 $\mu \mathrm{m}$, subglobosos, cruciado-septados; esterigmas hasta de $4 \mu \mathrm{m}$ de ancho. Basidiosporas de 10-12 × 8-9 $\mu \mathrm{m}$, elipsoides a ovadas, lisas, hialinas, gutuladas, apéndice hilar conspicuo.

Resumen taxonómico

Hábitat y hábito. Lignícola; crece sobre tallos de plantas herbáceas no identificadas, solitario.

Material estudiado. CAMPECHE: Loc. 2, Cappello 1009, 31-oct-2001 (FCME 18769); Loc. 7, Cappello 1194, 20-sep-2003 (FCME 20149); s/1, Valenzuela 10435, s/f (FCME 19229); Loc. 9, Sierra 1044, 29-oct-2002 (FCME 19537); Loc. 6, Cappello 1021, 01-nov-2001 (FCME 18752).

Comentarios taxonómicos. El género Tremelloscypha D. A. Reid fue colocado dentro de la familia Sebacinaceae K. Wells y Oberw. (Wells y Oberwinkler, 1982) junto con Sebacina Tul. y Tremellodendron G.F. Atk. (KisimovaHorovitz et al., 2000). Hasta el momento, sólo 2 especies se han descrito en el mundo: T. australiensis D. A. Reid y $T$. gelatinosa. Se les puede diferenciar por el tipo de sustrato en el que crecen, la talla de los basidiomas y el tamaño y forma de las basidiosporas, así como por su distribución. Tremelloscypha australiensis presenta basidiomas de 0.7-2 $\times 0.8-1.2 \mathrm{~mm}$, basidiosporas de 10.5$13 \times 5-5.5 \mu \mathrm{m}$ de cilíndricas a alantoides. T. gelatinosa ha sido citada para México de los estados de Quintana Roo y Yucatán (Guzmán, 2003; Guzmán, 2004). Es registro nuevo para el estado de Campeche.

Dacryopinax elegans (Berk. et M. A. Curtis) G. W. Martin, Lloydia 11:116 (1948) (Fig. 1C)

Basidioma de 10-36 mm de altura; pileado-estipitado; cartilaginoso-correoso, quebradizo en seco. Píleo de 7-22 mm de diámetro; cifeloide a campanulado, cocleariforme-flabeliforme, ejemplares maduros con el margen ondulado; color canela (6D6) a coñac-café óxido (6E78), hasta café rojizo (8E5-6). Estípite hasta de 1-4 mm de grosor; excéntrico, cilíndrico a aplanado; tomentoso; blanquecino a color canela (6D6) a café rojizo (8E5). Contexto menor a $1 \mathrm{~mm}$ de grosor. Himenio unilateral localizado en la parte interna de la copa; liso; color canela (6D6) a café obscuro (8E-F8). Probasidios y metabasidios de 48-55 $\times$ 4-5 $\mu \mathrm{m}$; cilíndricos, tendiendo a bifurcarse al madurar; esterigmas de 17-20 × $\mu \mathrm{m}$, atenuados en el ápice. Basidiosporas de 13-16 × 4-6 $\mu \mathrm{m}$, curvado-cilíndricas, aseptadas a triseptadas, septos muy engrosados, pared gruesa, lisas, hialinas, germinan produciendo conidios. Hifas del subhimenio con fíbulas. Pelos abhimeniales cilíndricos, septados, hialinos a amarillentos, de paredes muy gruesas, su contenido se tiñe con floxina.

\section{Resumen taxonómico}

Hábitat y hábito. Lignícola; sobre restos de madera no identificada, gregario.

Material estudiado. CAMPECHE: Loc. 6, Sierra 984, 1-nov-2001 (FCME 18734); Loc. 7, Sierra 1151, 6-sep2004 (FCME 20847); Loc. 8, Sierra 1167, 7-sep-2004 (FCME 20803).

Comentarios taxonómicos. Esta especie se distingue fácilmente por la coloración marrón-rojiza de los basidiomas y el contexto delgado, así como por la forma que presentan algunos ejemplares inmaduros (orbiculares o cocleariformes) y la septación (3) de las esporas. Sierra y Cifuentes (1993) describieron erróneamente como $D$. elegans una especie que actualmente es conocida como D. lowyi S. Sierra et Cifuentes (Sierra y Cifuentes, 2005), la cual presenta esporomas con el contexto más grueso, una zona de vesículas globosas o células en cadena en el subhimenio y su distribución es en zonas templadas a diferencia de $D$. elegans que principalmente es de regiones tropicales. Se ha registrado de los estados de Campeche, Morelos, Oaxaca, Puebla, Tabasco y Veracruz (Mendiola y Guzmán, 1973; Sierra, 1995, 2000).

Dacryopinax spathularia (Schwein.) G. W. Martin, Lloydia 11:116 (1948).

Basidioma de 8-10 mm de altura; cilíndrico a ligeramente capitado cuando joven a espatulado-flabeliforme o en forma de medio embudo cuando maduro; gelatinoso-cartilaginoso, no quebradizo; de color amarillo chabacano (5B6) en el ápice y café chocolate (6E6) hacia la base. Píleo 2-8 mm de ancho; estriado, piloso. Himenio unilateral, restringido a la zona superior no pilosa; liso o con estrías; anaranjado-grisáceo (5B5) a amarillo-oro (5B7) los más claros y obscureciéndose hacia anaranjado-café (6C6-6C8). Estípite de 1-2 mm de grosor; piloso; no quebradizo; la parte de unión al sustrato es blanquecina por la presencia de pelos. Probasidios de 25-27 $\times 3 \mu \mathrm{m}$, cilíndricos a subcilíndricos, hialinos, gutulados. Los metabasidios son de 24-29 ×3-4 $\mu \mathrm{m}$, de cilíndricos a subclavados, bifurcados, hialinos, gutulados; esterigmas de 10-15 × 2-4 $\mu \mathrm{m}$, cilíndricos, atenuados hacia el ápice, hialinos, gutulados. Basidiosporas de 9-11 $\times$ 4-6 $\mu \mathrm{m}$, faseoliformes, hialinas a amarillentas, lisas, gutuladas, no septadas a uniseptadas transversalmente. Pelos abhi- 
meniales, mayores de $100 \times 3-5 \mu \mathrm{m}$, septados de paredes muy gruesas, hialinos a amarillentos, su contenido se tiñe con floxina. Hifas sin fíbulas.

Resumen taxonómico

Hábitat y hábito. Lignícola; sobre restos de madera no identificada, gregario.

Material estudiado. CAMPECHE: Loc. 1, Sierra 991, 2-nov-2001 (FCME 18815).

Comentarios taxonómicos. Este ejemplar presenta forma flabelada por lo que inicialmente se pensó que podría tratarse de otra especie; sin embargo al hacer la revisión micromorfológica se llegó a la conclusión de que coincide con las descripciones de D. spathularia de Martin (1952), McNabb (1965), Lowy (1971) y Courtecuisse y Lowy (1990). Lowy (1971) mencionó que las variaciones morfológicas (flabeliforme vs. clavado) se presentan en las recolectas de zonas templadas (las primeras) o tropicales (las segundas), lo que ocasiona que los basidiomas presenten diferentes estados de los caracteres macroscópicos. Las características microscópicas son muy homogéneas, ya que las basidiosporas presentan solamente un septo y su zona abhimenial tiene pelos alargados, cilíndricos, de pared gruesa y septados. Se ha registrado en los estados de Campeche, Chiapas, Estado de México, Jalisco, Morelos, Michoacán, Nayarit, Oaxaca, Puebla, Quintana Roo, Veracruz y Valle de México (Chio y Guzmán, 1982; Sierra, 1995, 2000; Guzmán, 2003).

Auricularia cornea Ehrenb., Horae Phys. Berol.:91 (1820) (Fig. 1D)

Basidioma de hasta $60 \mathrm{~mm}$ de largo y $45 \mathrm{~mm}$ de alto; campanulado a ligeramente extendido, irregularmente lobulado; sésil; gelatinoso-cartilaginoso cuando fresco. Abhimenio hirsuto; de color rojizo (6C4), café piel o cognac (6E7-6), a gris casi blanco. Himenio liso; gris pardusco (6D2) a marrón obscuro (7F6). Contexto de $1 \mathrm{a} 2 \mathrm{~mm}$ de grosor; en 2 capas desprendibles; de color café-rojizo. Médula presente; de 413-498 $\mu \mathrm{m}$ de grosor, hifas de 3.1 a $4.2 \mu \mathrm{m}$, localizada en la parte central del contexto, arreglo hifal laxo. Hifas de 1.1 a $5.2 \mu \mathrm{m}$ de grosor dependiendo de la zona del contexto; presenta hifas anastomosadas y otras formando nudos; con fíbulas y septos globosos. Probasidios cilíndricos a subcilíndricos, hialinos; basidios de 55.1-69 $\times$ 4.2-5.5 $\mu \mathrm{m}$ con 3 septos transversales; esterigmas cilíndricos, lisos, hialinos. Dicariofisas, lisas, hialinas, a veces más grandes que los basidios. De una sola base surgen dicariofisas y basidios en una proporción 2:1. Basidiosporas de 14.8$16.9 \times 5.5-6.3 \mu \mathrm{m}$ alantoides, lisas, hialinas, germinación por repetición. Pelos abhimeniales de 80-400 ×5.5-8.5 $\mu \mathrm{m}$, rectos, ápice agudo, hialinos; base de los pelos hasta de $48 \mu \mathrm{m}$ de largo, radicante y simple, lisa, presenta una coloración marrón.

Resumen taxonómico

Hábitat y hábito. Lignícola; sobre restos de madera no identificada, gregario.

Material estudiado. CAMPECHE: Loc. 1, Sierra 1172, 09-sep-2004 (FCME 20740).

Comentarios taxonómicos. Puede confundirse con $A$. polytricha (Mont.) Sacc. la cual presenta una médula compacta y bien definida; el tamaño de los pelos de hasta $1000 \mu \mathrm{m}$ de largo y la base radicante de los mismos, de hasta $84 \mu \mathrm{m}$, son mucho mayores que los de A. cornea. Vistas de manera macromorfológica son muy parecidas y no es sino hasta la revisión micromorfológica cuando se delimitan las especies. Wong y Wells (1987) sostienen que son una misma especie, cuyo nombre más antiguo sería $A$. cornea. En el presente estudio a $A$. cornea y $A$. polytricha se consideran especies diferentes, con base principalmente en las características micromorfológicas de los ejemplares estudiados. Lowy $(1951,1952)$ describió la estructura de los basidiomas en las especies de este género, el cual es un carácter muy utilizado para la delimitación específica. Se ha registrado en los estados de Chiapas, Quintana Roo y Veracruz (Guzmán, 2003; Chanona-Gómez et al., 2007; Ruán-Soto et al., 2007). Es registro nuevo para el estado de Campeche.

Auricularia delicata (Mont.) Henn., Bot. Jb. 17:492 (1893) (Fig. 1E)

Basidioma de 20 a $145 \mathrm{~mm}$ de diámetro; pileado-sésil a subestipitado; gelatinoso-cartilaginoso cuando fresco; de tonos color marrón (6E6 y D5D). Píleo auriculiforme, ejemplares maduros con el margen ondulado; liso a poco sulcado, superficie velutinosa a vilosa. Himenio poroidereticulado. Contexto de 1 a $3 \mathrm{~mm}$ de grosor; en una sola capa, de color marrón claro. Médula presente. Hifas de 1 a $8.4 \mu \mathrm{m}$ de grosor, dependiendo de la zona del contexto; presenta hifas anastomosadas y otras formando nudos; con fíbulas, septos globosos y simples. Probasidios de 34.7-73.5 × 4.2-5.3 $\mu \mathrm{m}$, cilíndricos, lisos, hialinos, generalmente gutulados. Metabasidios de (34.7) $42-73.5 \times$ 3.2-5.3 $\mu \mathrm{m}$, con 3 septos transversales, cilíndricos, lisos, hialinos; esterigmas de 15.8-73.5 $\times$ 2.1-3.2 $\mu \mathrm{m}$, cilíndricos, lisos, hialinos. Basidiosporas de 11.6-13.7 ×4.2-6.3 $\mu \mathrm{m}$, alantoides a elipsoides, lisas, hialinas, generalmente gutuladas, germinación por repetición. Pelos del abhimenio de 26.3-157.5 ×3.2-6.3 $\mu \mathrm{m}$, rectos, ápice agudo, lisos, hialinos, base inflada a elongada con ramificación simple. Resumen taxonómico

Hábitat y hábito. Lignícola; sobre restos de madera no identificada, gregario. 
Material estudiado. CAMPECHE: Loc. 1, Sierra 1038, 28-oct-2002 (FCME 19516); Loc. 9, Villegas R. M. 2305, 29-oct-2002 (FCME 19447); Loc. 5, Villegas R. M. 2410, 22-sep-2003 (FCME 20184).

Comentarios taxonómicos. Esta especie es fácilmente distinguible por la presencia de retículo en la zona del himenio, el cual se observa tanto en ejemplares frescos como herborizados.

De manera macromorfológica, todos los ejemplares son casi homogéneos, con diferencia en cuanto a la cantidad de pelos del píleo; sin embargo, el carácter diagnóstico de esta especie (himenio reticulado) es homogéneo en cada uno de los ejemplares. Esto concuerda con las descripciones de Lowy $(1951 ; 1952 ; 1962 ; 1971)$ y Kobayasi (1981). Se ha registrado en los estados de Campeche, Chiapas, Hidalgo, Jalisco, Morelos, Oaxaca, Puebla, Sonora, Tabasco y Veracruz (Lowy, 1971, 1984; PérezSilva, 1971; Guzmán y García-Saucedo, 1973; Guzmán y Johnson, 1974; Frutis y Guzmán, 1983; Martínez-Alfaro et al., 1983; Portugal-Portugal et al., 1985; Pérez-Silva et al., 1992a; Herrera-Fonseca et al., 2002; Ruán-Soto et al., 2004, 2006, 2007; Cappello, 2006).

Auricularia mesenterica (Dicks.) Pers., Mycol. eur. (Erlanga) 1:97 (1822) (Fig. 1F)

Basidioma resupinado a efuso reflejo, con lóbulos libres, margen ondulado, cartilaginoso-correoso cuando fresco. Abhimenio tomentoso con zonas concéntricas alternadas distintivas. Himenio venoso; de color marrón medio a obscuro (5F5, 6E5, 7F3, 8F2, 8F5). Contexto de $>1$ a 1 $\mathrm{mm}$ de grosor; en 2 capas desprendibles; transparente. Sin médula. Hifas de 1.1 a $6.4 \mu \mathrm{m}$ de grosor dependiendo de la zona del contexto; presentan hifas anastomosadas y otras formando nudos; con fíbulas y septos globosos. Metabasidios de 45-55 ×3.2-4.5 $\mu \mathrm{m}$, con 3 septos transversales, cilíndricos, hialinos, lisos; esterigmas no observados. Basidiosporas de 12-16.5 ×5.5-6.4 $\mu \mathrm{m}$, alantoides, lisas, hialinas, germinación por repetición. Pelos abhimeniales de 375-870 × 2.5-3.5 $\mu \mathrm{m}$, rectos, ápice trunco, lisos, hialinos, se agrupan en mechones densos.

Resumen taxonómico

Hábitat y hábito. Lignícola; sobre restos de madera no identificada, gregario.

Material estudiado. CAMPECHE: Loc. 1, Sierra 1039, 28-oct-2002 (FCME 19526); Cappello 2056, 25-sep2003 (FCME 20174); González-Ávila 39, 9-nov-2005 (FCME 22021); Loc. 3, Sierra 1026, 26-oct-2002 (FCME 19496); Loc. 5, Villegas R. M. 2278, 25-oct-2002 (FCME 19430); Sierra 1035, 27-oct-2002 (FCME 19470); Loc. 6, Sierra 983, 1-nov-2001 (FCME 18812).

Comentarios taxonómicos. Esta especie es fácil de distinguir por el tipo de crecimiento resupinado a efuso-reflejo, así como por la presencia de zonas concéntricas en el abhimenio formadas por los pelos. Por la forma resupinada del basidioma de $A$. mesenterica, se confunde con el grupo de los teleforoides; sin embargo, los caracteres micromorfológicos son diferentes. Se ha registrado en los estados de Campeche, Chiapas, Durango, Guerrero, Hidalgo, Jalisco, Morelos, Oaxaca, Querétaro, Sonora, Tabasco y Veracruz (Lowy, 1971, 1980, 1984; Guzmán y García-Saucedo, 1973; Frutis y Guzmán, 1983; Pérez-Silva et al., 1992a; Herrera-Fonseca et al., 2002; Díaz-Moreno, 2005; Cappello, 2006; Landeros et al., 2006; Chanona-Gómez et al., 2007).

\section{Discusión}

El conocimiento taxonómico de los hongos gelatinosos es escaso en todo el mundo debido, entre otras razones, a los pocos investigadores que han centrado sus estudios en este grupo y lo esporádicamente que ha sido incluido en listados o micobiotas. Si se toma como referencia a Kirk et al. (2008), para la clase Tremellomycetes hay 377 especies citadas a escala mundial. Respecto al número de especies citadas para el país y como se menciona en la Introducción, son pocos los trabajos enfocados en este grupo y es necesaria una revisión exhaustiva de los ejemplares para corroborar su determinación. El presente trabajo aporta un incremento en la distribución de los hongos tremeloides, 2 nuevos registros para el estado de Campeche y el registro para la $\mathrm{RBC}$ de las 7 que se encontraron, aumentando así de 8 a 15 el número de especies fúngicas citadas hasta ahora para la reserva. Por otra parte, a pesar de que la RBC es la más grande de las áreas forestales protegidas del trópico mexicano y una de las 3 mayores de Mesoamérica, el estudio de la diversidad de la mayor parte de grupos de hongos todavía se encuentra en niveles muy bajos con respecto a extensiones más reducidas, como es el caso de las reservas de El Edén y Sian Ka'an. Haciendo un comparativo con respecto al número de especies presentes en la zona y en general para la península, Roberts (2008) cita, tan sólo de hongos Heterobasidiomycetes, 28 especies para Belice. Esto da un indicativo de la importancia de llevar a cabo un mayor número de proyectos y apoyar la elaboración de un inventario completo de la biodiversidad existente en la reserva y así poder rediseñar planes para su uso, manejo y conservación.

\section{Agradecimientos}

A los 2 revisores del manuscrito por sus atinadas observaciones y recomendaciones. El trabajo fue financiado por la UNAM-DGAPA IN-209605, IN-218008 e IN-207311. 


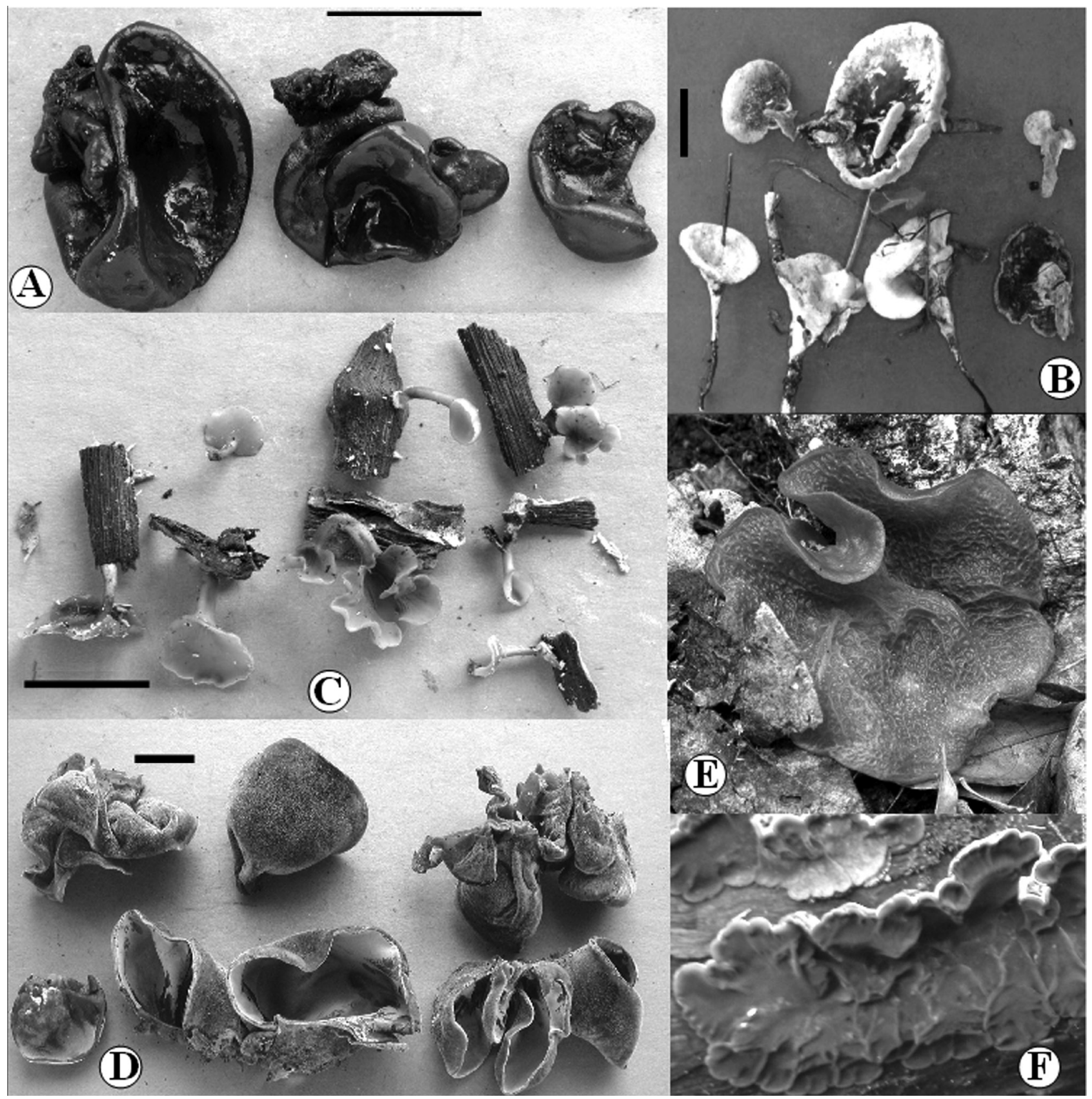

Figura 1. Basidiomas. A, Tremella wrightii; B, Tremelloscypha gelatinosa; C, Dacryopinax elegans; D, Auricularia cornea; E, Auricularia delicata; F, Auricularia mesenterica. Barras de referencia $=2 \mathrm{~cm}$. 


\section{Literatura citada}

Cappello, G. S. 2006. Hongos del Yumka'. Guía ilustrada. Universidad Juárez Autónoma de Tabasco/ Secretaría de Desarrollo Social y Protección al Medio Ambiente, Gobierno del Estado de Tabasco, Villahermosa, Tabasco. 105 p.

Chanona-Gómez F., R. Andrade, J. Castellanos y J. E. Sánchez. 2007. Macromicetos del Parque Laguna Bélgica, Municipio de Ocozocuautla de Espinoza, Chiapas, México. Revista Mexicana de Biodiversidad 78:369-381.

Chio, R. E. y G. Guzmán. 1982. Los hongos de la península de Yucatán, I. Las especies de macromicetos conocidas. Biotica 7:385-400.

Cifuentes, J. 1996. Estudio taxonómico de los géneros hidnoides estipitados (Fungi: Aphyllophorales) en México. Tesis, doctorado. Facultad de Ciencias, Universidad Nacional Autónoma de México, México, D. F. 289 p.

Cifuentes, J., M. Villegas y L. Pérez-Ramírez. 1986. Hongos. In Manual de herbario, A. Lot y F. Chiang (eds.). Consejo Nacional de la Flora de México, México, D. F. p. 55-64.

Courtecuisse, R. y B. Lowy. 1990. Elements for a mycological inventory of the vicinity of Saut Pararé (Arataye River) and Nouragues Inselberg (French Guiana). III. Heterobasidiomycetidae. Mycotaxon 39:329-344.

Díaz, R., J. Marmolejo y R. Valenzuela. 2005. Flora micológica de bosques de pino y pino-encino en Durango, México. Ciencia UANL 8:362-369.

Frutis, I. y G. Guzmán, 1983. Contribución al conocimiento de los hongos del estado de Hidalgo. Boletín de la Sociedad Mexicana de Micología 18:219-265.

García-Romero, L., G. Guzmán y T. Herrera. 1970. Especies de macromicetos citadas de México, I. Ascomycetes, Tremellales y Aphyllophorales. Boletín de la Sociedad Mexicana de Micología 4:54-76.

Guzmán, G. 1983. Los hongos de la península de Yucatán. II. Nuevas exploraciones y adiciones micológicas. Biotica 8:71100.

Guzmán, G. 1998. Inventorying the fungi in Mexico. Biodiversity and Conservation 7:369-384.

Guzmán, G. 2003. Los hongos de El Edén, Quintana Roo. Introducción a la micobiota tropical de México. CONABIO/ Instituto de Ecología, Xalapa, Veracruz. 316 p.

Guzmán, G. 2004. Los hongos de la Península de Yucatán (México). V. Nuevas observaciones y nuevos registros. Revista Mexicana de Micología 18:7-12.

Guzmán, G. y X. Madrigal. 1967. Notas sobre algunos hongos superiores de Escárcega, Campeche. Revista de la Sociedad Mexicana de Historia Natural 28:23-38.

Guzmán, G. y D. A. García-Saucedo. 1973. Macromicetos del estado de Jalisco. I. Consideraciones generales y distribución de las especies conocidas. Boletín de la Sociedad Mexicana de Micología 7:129-143.
Guzmán, G. y P. D. Johnson. 1974. Registros y especies nuevas de los hongos de Palenque, Chiapas. Boletín de la Sociedad Mexicana de Micología 8:73-105.

Hawksworth, D. L. 1991. The fungal dimension of biodiversity: magnitude, significance and conservation. Mycological Research 95:641-655.

Hawksworth, D. L. 1997. Fungi and international biodiversity initiatives. Biodiversity and Conservation 6:661-668.

Hawksworth, D. L., P. M. Kirk, B. C. Sutton y D. N. Pegler. 1995. Dictionary of the Fungi. International Mycological Institute, CAB International, Wallingford. 650 p.

Herrera-Fonseca, M. J., L. Guzmán-Dávalos y O. Rodríguez. 2002. Contribución al conocimiento de la micobiota de la región de San Sebastián del Oeste, Jalisco, México. Acta Botanica Mexicana 58:19-50.

Herrera, T., E. Pérez-Silva, M. Esqueda y V. H. Valenzuela. 2005. Algunos gasteromicetos de Calakmul, Campeche, México. Revista Mexicana de Micología 21:23-27.

INE (Instituto Nacional de Ecología). 1999. Programa de Manejo de la Reserva de la Biosfera Calakmul, México, D. F. 272 p.

Kirk, P. M., P. F. Cannon, D. W. Minter y J. A. Stalpers. 2008. Dictionary of the fungi, décima edición. CAB International, Wallingford. $784 \mathrm{p}$.

Kisimova-Horovitz, L., F. Oberwinkler y L. D. Gómez. 2000. Basidiomicetos resupinados de Costa Rica. Myxariaceae Jülich, Sebacinaceae Wells \& Oberw., y Tremellodendropsidaceae Jülich. Revista de Biología Tropical 48:519-538.

Kobayasi, Y. 1981. The genus Auricularia. Bulletin of the National Science Museum, Tokyo, Series B 7:41-67.

Kornerup, A. y J. H. Wanscher. 1978. Methuen handbook of colour. Eyre, London. 252 p.

Küppers, H. 1979. Atlas de los colores. Blume, Barcelona. 161 p.

Landeros, F., J. Castillo, G. Guzmán y J. Cifuentes. 2006. Los hongos (macromicetos) conocidos en el cerro el Zamorano (Querétaro-Guanajuato), México. Revista Mexicana de Micología 22:25-31.

Lowy, B. 1951. A morphological basis for classifying the species of Auricularia. Mycologia 43:351-358.

Lowy, B. 1952. The genus Auricularia. Mycologia 44:656-692.

Lowy, B. 1962. Contribución al estudio de los Tremellales de la Argentina. Lilloa 31:212-228.

Lowy, B. 1965. Estudio sobre algunos Tremellales de México. Boletín de la Sociedad Botánica de México 29:19-33.

Lowy, B. 1971. Flora Neotropica, Monograph 6. Tremellales. Hafner, Nueva York. 153 p.

Lowy, B. 1980. Tremellales. Flora Neotropica 6 (Suppl.):1-18.

Lowy, B. y G. Guzmán. 1979. Nuevos registros de Tremellales de México. Boletín de la Sociedad Mexicana de Micología 13:211-214.

Martin, G. W. 1952. Revision of the North Central Tremellales. J. Cramer, Lehre. 122 p. 
Martínez-Alfaro, M. A., E. Pérez-Silva y E. Aguirre-Acosta. 1983. Etnomicología y exploraciones micológicas en la sierra Norte de Puebla. Boletín de la Sociedad Mexicana de Micología 18:51-63.

Munsell Color. 1975. Munsell soil color chart. United States Department of Agriculture, Kollmorgen, Maryland. 34 p.

McNabb, R. F. R. 1965. Taxonomic studies in the Dacrymycetaceae. III. Dacryopinax. New Zealand Journal of Botany 3:59-72.

Mendiola, G. y G. Guzmán. 1973. Las especies de Tremellales conocidas en México. Boletín de la Sociedad Mexicana de Micología 7:89-97.

Pérez-Silva, E. 1971. Algunos macromicetos de Chiapas, México. Boletín de la Sociedad Mexicana de Micología 5:23-35.

Pérez-Silva, E., T. Herrera Suárez y R. Valenzuela. 1992. Hongos (Macromicetos) de la península de Yucatán. In Diversidad biológica en la Reserva de la Biosfera de Sian Ka'an, Quintana Roo, México, volumen II. D. Navarro y E. SuárezMorales (eds.). CIQRO/ SEDESOL, Chetumal, Quintana Roo. p. 13-22.

Pérez-Silva, E., T. Herrera y M. Esqueda-Valle. 1992a. First records of jelly fungi (Dacrymycetaceae, Auriculariaceae, Tremellaceae) from Sonora, Mexico. Mycotaxon 44:475483.

Portugal, D., E. Montiel, L. López-Eustaquio y V. Mora. 1985. Contribución al conocimiento de los hongos que crecen en la región de El Texcal, estado de Morelos. Revista Mexicana de Micología 1:401-412.

Roberts, P. 2008. Heterobasidiomycetes from Belize. Kew Bulletin 63:87-99.

Roberts, P. y A. de Meijer. 1997. Macromycetes from Paraná State, Brazil 7: Sirobasidiaceae and Tremellaceae. Mycotaxon 64:261-284.

Ruan-Soto, F., R. Garibay-Orijel y J. Cifuentes. 2004. Conocimiento micológico tradicional en la planicie costera del Golfo de México. Revista Mexicana de Micología 19:5770 .

Ruan-Soto, F., R. Garibay-Orijel y J. Cifuentes. 2006. Process and dynamics of traditional selling wild edible mushrooms in tropical Mexico. Journal of Ethnobiology and Ethnomedicine 2:1-13

Ruán-Soto, F., R. Mariaca, J. Cifuentes, F. Limón, L. PérezRamírez y S. Sierra. 2007. Nomenclatura, clasificación y percepciones locales acerca de los hongos en dos comunidades de la Selva Lacandona, Chiapas, México. Etnobiología 5:1-20.

Ruán-Soto, F., J. Cifuentes, R. Mariaca, F. Limón, L. PérezRamírez y S. Sierra. 2009. Uso y manejo de hongos silvestres en dos comunidades de la selva lacandona, Chiapas, México. Revista Mexicana de Micología 29:61-72.

Sierra, S. 1992. Contribución al estudio taxonómico de algunos hongos tremeloides (Heterobasidiomycetes) del centro y sur de México. Tesis, Facultad de Ciencias, Universidad Nacional Autónoma de México, México, D. F. 104 p.

Sierra, S. 1995. Taxonomía y ultraestructura de algunas especies de la familia Dacrymycetaceae en México. Tesis, maestría. Facultad de Ciencias, Universidad Nacional Autónoma de México, México D. F. 110 p.

Sierra, S. 2000. Estudio taxonómico y monográfico del orden Dacrymycetales (Fungi) en México. Tesis, doctorado Facultad de Ciencias, Universidad Nacional Autónoma de México, México, D. F. 144 p.

Sierra, S. y J. Cifuentes. 1993. Contribución al estudio taxonómico de los hongos tremeloides (Heterobasidiomycetes) de México. Revista Mexicana de Micología 9:119-137.

Sierra, S. y J. Cifuentes. 1998. A new species of Phyllogloea from Mexico. Mycotaxon 66:501-508.

Sierra, S. y J. Cifuentes. 2005. A new species of Dacryopinax from Mexico. Mycotaxon 92: 243-250.

Sierra, S. y J. Cifuentes. 2009. Primer registro del género Cerinomyces (Fungi: Dacrymycetales) en México. Revista Mexicana de Biodiversidad 80:853-855.

Sierra, S., J. Cifuentes, F. Ruán-Soto y R. Mariaca. 2008. A white form of Auricularia fuscosuccinea from Lacandonia tropical forest, Chiapas, Mexico. Mycotaxon 105:415-419.

Wells, K. y F. Oberwinkler. 1982. Tremelloscypha gelatinosa, a species of a new family Sebacinaceae. Mycologia $74: 325-331$. 